\title{
WEAK SUBGROUPOID LATTICES
}

\author{
Alfonz Haviar - Miroslav Haviar \\ Dedicated to the 90th birthday of Professor Ján Jakubik \\ (Communicated by Miroslav Ploščica)
}

\begin{abstract}
The aim of the paper is a characterization of the lattice of all weak subgroupoids of a partial groupoid. It also extends to arbitrary finite algebras Pióro's result saying that the weak subgroupoid lattice of a finite commutative groupoid $G$ in which $g \cdot h \neq g$ (for all $g, h \in G$ ) uniquely determines its subgroupoid lattice.
\end{abstract}

(C) 2014

Mathematical Institute

Slovak Academy of Sciences

\section{Introduction}

W. Bartol [2] completely characterized the lattices of weak subalgebras of partial algebras. These are distributive algebraic lattices with additional properties concerning their join-irreducible elements. He also showed that for two unary algebras of arbitrary unary types their weak subalgebra lattices are isomorphic if and only if their undirected graphs (representing operations) are isomorphic.

K. Pióro [7 characterized lattices of all weak subgroupoids of partial finite commutative groupoids satisfying $g \cdot h \neq g$ for all elements $g, h$. To do this he used his results from [5] concerning directed hypergraphs. In [7] he further showed that the weak subgroupoid lattices of finite commutative groupoids satisfying $g \cdot h \neq g$, for all elements $g, h$, uniquely determine their subgroupoid lattices. We extend this result first to arbitrary finite groupoids, and then even to arbitrary finite algebras. For locally finite unary algebras of finite unary type the result was proved by Pióro in [4]. The same author then studied the weak subalgebra lattices for a given infinite unary type in the present journal in [6].

2010 Mathematics Subject Classification: Primary 08A55; Secondary 06B15.

Keywords: weak subalgebra, weak subgroupoid.

The second author acknowledges support from Slovak grants APVV-0223-10 and VEGA $1 / 0212 / 13$. 
Our focus is on a characterization of the lattices of weak subgroupoids. Only the last theorem of the paper is devoted to the lattices of weak subalgebras of finite partial algebras where we use some Bartol's results [2]. We first recall terminology and some results of [2]. Then we switch to the theory of partial groupoids.

We shall study partial algebras $\mathcal{A}=\left(A ;\left(f_{t}^{(A)}\right)_{t \in T}\right)$ of type $\tau=\left(\left(f_{t}\right)_{t \in T}\right)$ where $A$ is a universe of $\mathcal{A}$ and $f_{t}^{(A)}$ is an $n\left(f_{t}\right)$-ary partial operation on $A$, for each $t \in T$. We shall write briefly $\left(A ; f_{1}^{(A)}, \ldots, f_{n}^{(A)}\right)$ when $\tau=\left(f_{1}, \ldots, f_{n}\right)$ or simply $\left(A ; f_{1}, \ldots, f_{n}\right)$ when no confusion arises.

Definition 1.1. ([2]) A partial algebra $\mathcal{B}=\left(B ;\left(f_{t}^{(B)}\right)_{t \in T}\right)$ is called a weak subalgebra of $\mathcal{A}=\left(A ;\left(f_{t}^{(A)}\right)_{t \in T}\right)$ if $B \subseteq A$ and $f_{t}^{(B)} \subseteq f_{t}^{(A)}$ for each $t \in T$.

The set of all weak subalgebras of a partial algebra $\mathcal{A}$ will be denoted by $S_{w}(\mathcal{A})$. It not hard to see that $\left(S_{w}(\mathcal{A}), \leq\right)$ with the usual ordering is a complete lattice. The following basic observations of [2] say more.

Lemma 1.2. ([2] Let $\mathcal{A}=\left(A ;\left(f_{t}^{(A)}\right)_{t \in T}\right)$ be a partial algebra.

(a) The lattice $\left(S_{w}(\mathcal{A}), \leq\right)$ of all weak subalgebras of $\mathcal{A}$ is a distributive algebraic lattice with the following supremum and infimum operations: for any family of weak subalgebras $\mathcal{B}_{i}=\left(B_{i} ;\left(f_{t}^{\left(B_{i}\right)}\right)_{t \in T}\right)(i \in I)$ of $\mathcal{A}$,

$$
\begin{aligned}
& \bigvee_{i \in I} \mathcal{B}_{i}=\left(\bigcup_{i \in I} B_{i} ;\left(\bigcup_{i \in I} f_{t}^{\left(\mathcal{B}_{i}\right)}\right)_{t \in T}\right), \\
& \bigwedge_{i \in I} \mathcal{B}_{i}=\left(\bigcap_{i \in I} B_{i} ;\left(\bigcap_{i \in I} f_{t}^{\left(\mathcal{B}_{i}\right)}\right)_{t \in T}\right) .
\end{aligned}
$$

(b) $A$ weak subalgebra $\mathcal{B}=\left(B,\left(f_{t}^{(B)}\right)_{t \in T}\right)$ is an atom of the lattice $S_{w}(\mathcal{A})$ if and only if $B=\{a\}$ for an element $a \in A$ and $f_{t}^{(B)}=\emptyset$ for all $t \in T$.

(c) $A$ weak subalgebra $\mathcal{B}=\left(B,\left(f_{t}^{(B)}\right)_{t \in T}\right)$ of $\mathcal{A}$ is a join-irreducible element of $S_{w}(\mathcal{A})$ if and only if $\mathcal{B}$ is the least element of $S_{w}(\mathcal{A})$ or $\mathcal{B}$ is an atom or there are elements $f_{t}, t \in T$, and $\left[a_{1}, \ldots, a_{n\left(f_{t}\right)}, a\right] \in f_{t}^{(A)}$ such that $B=\left\{a_{1}, \ldots, a_{n\left(f_{t}\right)}, a\right\}, f_{t}^{(B)}=\left\{\left[a_{1}, \ldots, a_{n\left(f_{t}\right)}, a\right]\right\}$ and $f_{t^{\prime}}^{(B)}=\emptyset$ for all $t^{\prime} \in T \backslash\{t\}$.

(d) A weak subalgebra $\mathcal{B}$ is completely join-irreducible in the lattice $S_{w}(\mathcal{A})$ if and only if it is join-irreducible.

(e) Every element in $S_{w}(\mathcal{A})$ is a join of completely join-irreducible elements. 


\section{Remark 1.3.}

(i) While the least element of a lattice is usually not considered to be joinirreducible, we respect Bartol's choice in (c) above as the matter of a preference.

(ii) Consider a weak subalgebra $\mathcal{B}=\left(B,\left(f_{t}^{(B)}\right)_{t \in T}\right)$ where $f_{t}^{(B)} \neq \emptyset$ for at least two distinct elements $t_{1}, t_{2} \in T$. Then $\mathcal{B}$ can be in $S_{w}(\mathcal{A})$ expressed as the join of the weak subalgebras $\mathcal{B}_{1}=\left(B,\left(f_{t}^{\left(B_{1}\right)}\right)_{t \in T}\right)$ and $\mathcal{B}_{2}=\left(B,\left(f_{t}^{\left(B_{2}\right)}\right)_{t \in T}\right)$ where $f_{t_{1}}^{\left(B_{1}\right)}=\emptyset$ and $f_{t}^{\left(B_{1}\right)}=f_{t}^{(B)}$ for all $t \in T \backslash\left\{t_{1}\right\}$ while $f_{t_{2}}^{\left(B_{2}\right)}=\emptyset$ and $f_{t}^{\left(B_{2}\right)}=f_{t}^{(B)}$ for all $t \in T \backslash\left\{t_{2}\right\}$. Hence such a weak subalgebra $\mathcal{B}$ cannot be join-irreducible.

(iii) A weak subalgebra $\mathcal{B}=\left(B,\left(f_{t}^{(B)}\right)_{t \in T}\right)$ with $|B| \geq 2$ and $f_{t}^{(B)}=\emptyset$ for all $t \in T$ can be in $S_{w}(\mathcal{A})$ expressed as the join of the atoms $\left(\{b\},\left(f_{t}^{(\{b\})}\right)_{t \in T}\right)$ where $b \in B$ and $f_{t}^{(\{b\})}=\emptyset$ for all $t \in T$. Hence such a weak subalgebra cannot be join-irreducible, too.

The proof of the following lemma is straightforward.

Lemma 1.4. A weak subalgebra $\mathcal{D}=\left(D,\left(f_{t}^{(\mathcal{D})}\right)_{t \in T}\right)$ of $\mathcal{A}$ covers a weak subalgebra $\mathcal{B}=\left(B,\left(f_{t}^{(\mathcal{B})}\right)_{t \in T}\right)$ of $\mathcal{A}$ in the lattice $\left(S_{w}(\mathcal{A}), \leq\right)$ if and only if one of the following conditions is satisfied:

(a) $D=B \cup\{a\}$ for some element $a \in A \backslash B$ and $f_{t}^{(D)}=f_{t}^{(B)}$ for each element $t \in T$;

(b) $D=B$ and there are $t \in T$ and elements $a_{1}, \ldots, a_{n\left(f_{t}\right)}, a \in A$ for which $f_{t}^{(D)}=f_{t}^{(B)} \cup\left\{\left[a_{1}, \ldots, a_{n\left(f_{t}\right)}, a\right]\right\}$, where $\left[a_{1}, \ldots, a_{n\left(f_{t}\right)}, a\right] \notin f_{t}^{(B)}$, and $f_{t^{\prime}}^{(B)}=f_{t^{\prime}}^{(D)}$ for all $t^{\prime} \in T \backslash\{t\}$.

\section{Weak subgroupoid lattices}

Let $\mathcal{G}=(G, f)$ be a partial groupoid. We shall sometimes write $[a, b, c] \in f$ instead of $f(a, b)=c$. The lattice of all weak subgroupoids of a partial groupoid $\mathcal{G}$ will be denoted by $S_{w}(\mathcal{G})$. We shall assume that the considered groupoids are non-trivial.

From Lemma 1.2(d) it follows that every join-irreducible element of $S_{w}(\mathbf{G})$ is completely join-irreducible. The next lemma describes the (completely) joinirreducible elements in the lattice $S_{w}(\mathcal{G})$ of all weak subgroupoids of a partial non-trivial groupoid $\mathcal{G}$. 
Lemma 2.1. The lattice $S_{w}(\mathcal{G})$ of all weak subgroupoids of a non-trivial partial groupoid $\mathcal{G}=(G, f)$ contains (completely) join-irreducible elements of (at most) four types:

(T0) the least element $(\emptyset, \emptyset)$ and the atoms $(\{a\}, \emptyset)$, for $a \in G$;

(T1) $(\{a\},\{[a, a, a]\})$ provided $f(a, a)=a$, for $a \in G$;

(T2) $(\{a, b\},\{[a, a, b]\})$ provided $f(a, a)=b$ or $(\{a, b\},\{[a, b, f(a, b)]\})$ provided $f(a, b) \in\{a, b\}$, for distinct $a, b \in G$;

(T3) $(\{a, b, c\},\{[x, y, z]\})$ provided $f(x, y)=z$, for distinct $a, b, c \in G$ and distinct $x, y, z \in\{a, b, c\}$ in case $|G| \geq 3$.

P r o of. From Lemma 1.2 it follows that a (completely) join-irreducible element of $S_{w}(\mathcal{G})$ is either the least element $(\emptyset, \emptyset)$ or the atom $(\{a\}, \emptyset)$, for $a \in G$, or a weak subgroupoid $\mathcal{G}^{\prime}=\left(G^{\prime}, f^{\left(G^{\prime}\right)}\right)$ such that $G^{\prime}=\{a, b, c\}$ and $f^{\left(G^{\prime}\right)}=\{[x, y, z]\} \quad$ for $\quad x, y, z \in\{a, b, c\}$ and $a, b, c \in G$.

Let us consider the latter case. The equality $a=b=c$ leads to the type (T1) while the subcases $|\{a, b, c\}|=2$ and $|\{a, b, c\}|=3$ lead to the types (T2) and (T3), respectively.

Definition 2.2. Let $\mathcal{L}=(L ; \leq)$ be an algebraic distributive lattice. We shall denote:

(i) by $A(L)$ the set of all atoms of $\mathcal{L}$;

(ii) by $I(L)$ the set of all join-irreducible elements of $\mathcal{L}$ covering the atoms or joins of pairs of atoms;

(iii) by $J(L)$ the set of all join-irreducible elements of $\mathcal{L}$ covering joins of triples of atoms $p \vee q \vee r$, where $p, q, r$ are mutually distinct;

(iv) by $S_{i}, i \in I(L)$, the set $\{[a, b] \in A(L) \times A(L) \mid a<i \& b<i\}$;

(v) by $S_{j}, j \in J(L)$, the set

$$
\{[a, b] \in A(L) \times A(L) \mid a<j \& b<j \& a \neq b\} .
$$

\section{Remark 2.3.}

a) With respect to Definition 2.2 we firstly note a well-known and easily seen fact that in a distributive lattice $L$, the join of a finite number of atoms cannot be above any other atom (that is, $a \leq \bigvee S$ is impossible for any finite $S \subseteq A(L)$ and $a \in A(L) \backslash S)$.

b) We further note that if $\mathcal{L}=(L ; \leq)$ is taken to be the lattice $S_{w}(\mathcal{G})$ of all weak subgroupoids of a non-trivial partial groupoid $\mathcal{G}=(G, f)$, then from the description of the (completely) join-irreducible elements of $S_{w}(\mathcal{G})$ in Lemma 2.1 we have that: 
(i) $A(L)$ is the set of non-zero (completely) join-irreducible elements of the type (T0);

(ii) $I(L)$ is the set of (completely) join-irreducible elements of the types (T1) or $(\mathrm{T} 2)$;

(iii) $J(L)$ is the set of (completely) join-irreducible elements of the type (T3);

(iv) there are finitely many atoms below each (completely) join-irreducible element $k \in I(L) \cup J(L)$, and hence for all $k \in I(L) \cup J(L)$, the sets $S_{k}$ are finite;

(v) for an illustration, the element $(\{a, b, c\},\{[a, b, a]\})$ of $S_{w}(\mathcal{G})$, where $a, b, c \in G$ are distinct and $f(a, b)=a$, is not join-irreducible since it can be expressed as $(\{a, b, c\},\{[a, b, a]\})=(\{a, b\},\{[a, b, a]\}) \vee(\{c\}, \emptyset)$.

Now we present the main result of this section.

TheOREM 2.4. An algebraic lattice $\mathcal{L}=(L ; \leq)$ is isomorphic to the lattice $S_{w}(\mathcal{G})$ for some partial groupoid $\mathcal{G}=(G, f)$ if and only if $\mathcal{L}$ is distributive and the next three conditions are satisfied:

(1) every element of $\mathcal{L}$ is a join of completely join-irreducible elements;

(2) every non-zero and non-atomic completely join-irreducible element covers either an atom or a join of a pair of atoms or a join of a triple of atoms;

(3) $|S| \leq\left|\bigcup_{k \in S} S_{k}\right|$ for every nonempty finite subset $S \subseteq I(L) \cup J(L)$.

Proof. The lattice $S_{w}(\mathcal{G})$ is algebraic and distributive according to Lemma 1.2(a).

Based on the above lemmas and remarks, it has the following properties:

a) For all $a \in G$, the weak subgroupoids $(\{a\}, \emptyset)$ are atoms of $S_{w}(\mathcal{G})$ and there are no other atoms.

b) For all $a \in G$, if $f(a, a)=a$, then the atom $(\{a\}, \emptyset)$ is covered in $S_{w}(\mathcal{G})$ by a join-irreducible element of the type (T1), namely the weak subgroupoid $(\{a\},\{[a, a, a]\})$; otherwise the atom $(\{a\}, \emptyset)$ is not covered by a join-irreducible element. (We note that an element $(\{a, x\}, \emptyset)$ covering, for any $x \in G$, the atom $(\{a\}, \emptyset)$ by Lemma 1.4 can be expressed as $(\{a, x\}, \emptyset)=(\{a\}, \emptyset) \vee(\{x\}, \emptyset)$, and so it is not join-irreducible.)

c) For all distinct $a, b \in G$, the join $(\{a, b\}, \emptyset)$ of the atoms $(\{a\}, \emptyset)$ and $(\{b\}, \emptyset)$ in $S_{w}(\mathcal{G})$ is covered by at most four join-irreducible elements $(\{a, b\}$, $\{[a, a, b]\}),(\{a, b\},\{[b, b, a]\}),(\{a, b\},\{[a, b, f(a, b)]\}),(\{a, b\},\{[b, a, f(b, a)]\})$ of the type (T2). (We note that an element $(\{a, b, x\}, \emptyset)$ covering, for any $x \in G$, the element $(\{a, b\}, \emptyset)$ by Lemma 1.4, can be expressed as $(\{a, b, x\}, \emptyset)=$ $(\{a, b\}, \emptyset) \vee(\{x\}, \emptyset)$. 
d) For all distinct $a, b, c \in G$, the join $(\{a, b, c\}, \emptyset)$ of the atoms $(\{a\}, \emptyset)$, $(\{b\}, \emptyset),(\{c\}, \emptyset)$ in $S_{w}(\mathcal{G})$ is covered by at most six join-irreducible elements of the type (T3).

Assume that $\mathcal{L}=(L ; \leq)$ is the lattice $S_{w}(\mathcal{G})$ for some partial groupoid $\mathcal{G}=(G, f)$. The above properties yield that the condition (2) is satisfied in the lattice $\mathcal{L}$. The condition (1) follows straightly from Lemma 1.2(e). The condition (3) follows from the fact that the (completely) join-irreducible elements $(\{a, b, c\},\{[x, y, z]\})$ in $I(L) \cup J(L)$ (where $x, y, z \in\{a, b, c\}$ and $f(x, y)=z)$ are of one of the types (T1) or (T2) or (T3), and for every non-empty finite subset $S \subseteq I(L) \cup J(L)$ the mapping

$$
\varphi: S \rightarrow \bigcup_{k \in S} S_{k}, \quad \varphi((\{a, b, c\},\{[x, y, z]\}))=[x, y]
$$

is one-to-one. Indeed, assume that in $S \subseteq I(L) \cup J(L)$,

$$
(\{a, b, c\},\{[x, y, z]\}) \neq\left(\left\{a^{\prime}, b^{\prime}, c^{\prime}\right\},\left\{\left[x^{\prime}, y^{\prime}, z^{\prime}\right]\right\}\right) .
$$

Since $z=f(x, y), z^{\prime}=f\left(x^{\prime}, y^{\prime}\right)$, the equality $[x, y]=\left[x^{\prime}, y^{\prime}\right]$ would lead to $z=z^{\prime}$, and thus to $[x, y, z]=\left[x^{\prime}, y^{\prime}, z^{\prime}\right]$; because we have that $x, y, z \in\{a, b, c\}$ and $x^{\prime}, y^{\prime}, z^{\prime} \in\left\{a^{\prime}, b^{\prime}, c^{\prime}\right\}$, it leads to the equality $\{a, b, c\}=\left\{a^{\prime}, b^{\prime}, c^{\prime}\right\}$, which finally gives $(\{a, b, c\},\{[x, y, z]\})=\left(\left\{a^{\prime}, b^{\prime}, c^{\prime}\right\},\left\{\left[x^{\prime}, y^{\prime}, z^{\prime}\right]\right\}\right)$, a contradiction. Hence $[x, y] \neq\left[x^{\prime}, y^{\prime}\right]$ as required.

Now let $\mathcal{L}=(L ; \leq)$ be a distributive algebraic lattice satisfying (1)-(3). Then there are only finitely many atoms below each (completely) join-irreducible element $k \in I(L) \cup J(L)$, and hence for all $k \in I(L) \cup J(L)$, the sets $S_{k}$ are finite. We shall define a partial groupoid $\mathcal{G}=(G, f)$ such that $\mathcal{L} \cong S_{w}(\mathcal{G})$.

Let $G=A(L)$. By the Hall theorem [3] and by the condition (3), for every finite subset $S \subseteq I(L) \cup J(L)$ there is an injective mapping $g: S \rightarrow \bigcup\left(S_{k}, k \in S\right)$ such that $g(k) \in S_{k}$, for every $k \in S$. Since all the sets $S_{k}$ are finite, by the Compactness Principle [1] there exists an injective mapping

$$
h: I(L) \cup J(L) \rightarrow \bigcup\left\{S_{k} \mid k \in I(L) \cup J(L)\right\}
$$

such that $h(k) \in S_{k}$ for every $k \in I(L) \cup J(L)$.

The partial operation $f$ on the set $G$ is determined as follows:

(i) $f(a, a)=a$ if there exists a join irreducible element $i \in I(L) \cup J(L)$ covering the atom $a$ and $h(i)=[a, a]$;

(ii) $f(a, a)=b$ if there exists a join-irreducible element $i \in I(L) \cup J(L)$ covering the join $a \vee b$ of atoms $a, b$ and $h(i)=[a, a]$;

(iii) $f(a, b)=a$ if there exists a join-irreducible element $i \in I(L) \cup J(L)$ covering the join $a \vee b$ of atoms $a, b$ and $h(i)=[a, b]$;

(iv) $f(a, b)=c$ if there exists a join-irreducible element $i \in I(L) \cup J(L)$ covering the join $a \vee b \vee c$ of distinct atoms $a, b, c$ and $h(i)=[a, b]$. 
In other cases the value $f(a, b)$ is not defined.

We shall use the notation $[a, b, c]_{j} \in f$ in case we want to emphasize that $f(a, b)=c$ is obtained from the existence of a join-irreducible element $j$ which covers $a \vee b \vee c$ for the atoms $a, b, c$, and $h(j)=[a, b]$.

We note that our hypotheses yield the following properties of $\mathcal{L}$ :

- every atom is covered by at most one join-irreducible element;

- the join $a \vee b$ of distinct atoms is covered by at most four join irreducible elements;

- the join $a \vee b \vee c$ of distinct atoms is covered by at most six join irreducible elements (we note that the existence of $S=\left\{j_{1}, j_{2}, \ldots, j_{7}\right\} \subseteq I(L) \cup J(L)$ with distinct $j_{\ell}$ covering the join $a \vee b \vee c$ would contradict $|S| \leq\left|\bigcup_{k \in S} S_{k}\right|$ since $\left.S_{j_{1}}=S_{j_{2}}=\cdots=S_{j_{7}}=\{[a, b],[a, c],[b, a],[b, c],[c, a],[c, b]\}\right)$.

We now define a mapping $\phi: L \rightarrow S_{w}(\mathcal{G})$ as follows:

$$
\phi(x)=\left(A_{x}, f_{x}\right), \quad \text { where } \quad A_{x}=\{a \in A(L) \mid a \leq x\}
$$

and for (not necessarily distinct) $a, b, c \in A(L)=G,[a, b, c]_{j} \in f_{x}$ iff $j \leq x$, $j \in I(L) \cup J(L)$, that is, $f(a, b)=c$ (the latter always meaning the existence of a join irreducible element $j \leq x$ for which $h(j)=[a, b])$.

To illustrate this, we note that $\phi(0)=(\emptyset, \emptyset), \phi(a)=(\{a\}, \emptyset)$ and $\phi(a \vee b)=$ $(\{a, b\}, \emptyset)$ if $a, b$ are atoms of $\mathcal{L}, \phi(i \vee j)=(\{a, b\},\{[a, b, a],[b, a, b]\})$ if $i, j$ are join-irreducible elements covering the join $a \vee b$ of distinct atoms $a, b$ and $h(i)=[a, b]$ and $h(j)=[b, a]$, etc.

We shall show that the mapping $\phi$ is an isomorphism.

Claim 1: $\phi$ is injective.

Let $x \neq y, x, y \in L$. Obviously $\phi(x) \neq \phi(y)$ in case there exists an atom $p \in\left(A_{x} \backslash A_{y}\right) \cup\left(A_{y} \backslash A_{x}\right)$. In the case $A_{x}=A_{y}$ there exists a join-irreducible non-atomic element $j \in I(L) \cup J(L)$ for which either $j \leq x$ and $j \not \leq y$ or $j \leq y$ and $j \not \leq x$. Again we get $\phi(x) \neq \phi(y)$ because we either have $[a, b, c]_{j} \in f_{x}$ and $[a, b, c]_{j} \notin f_{y}$, or $[a, b, c]_{j} \in f_{y}$ and $[a, b, c]_{j} \notin f_{x}$, where $f(a, b)=c$.

Claim 2: $\phi$ is surjective.

Let $\left(H, f^{*}\right)$ be a week subgroupoid of the partial groupoid $\mathcal{G}$. Let us denote:

$$
J_{0}:=\left\{j \in I(L) \cup J(L) \mid(\exists a, b, c)\left([a, b, c]_{j} \in f^{*}\right)\right\} .
$$

Let $z=\bigvee\left(H \cup J_{0}\right)$. We shall show that $\phi(z)=\left(H, f^{*}\right)$, that is, $A_{z}=H$ and $f_{z}=f^{*}$.

It is obvious that $H \subseteq A_{z}$ and $f^{*} \subseteq f_{z}$. Now let $p \in A_{z}$. Since $\mathcal{L}$ is algebraic and distributive, we get

$$
p=p \wedge z=p \wedge \bigvee\left(H \cup J_{0}\right)=\bigvee\left\{p \wedge x \mid x \in H \cup J_{0}\right\}
$$


and so $p=p \wedge x$ for $x \in H$ or $p=p \wedge x$ for $x \in J_{0}$. In the first case $p=x \in H$, in the latter case $p \leq j$ for $j \in I(L) \cup J(L)$, hence again $p \in H$. We conclude $H=A_{z}$.

Let $j \in I(L) \cup J(L), j \leq z$. Similarly as in the previous case it follows that

$$
j=j \wedge z=j \wedge \bigvee\left(H \cup J_{0}\right)=\bigvee\left\{j \wedge x \mid x \in H \cup J_{0}\right\}
$$

which gives $j \in J_{0}$ (because $j$ is completely join-irreducible). Consequently we conclude $f^{*}=f_{z}$.

Claim 3: $\phi$ and $\phi^{-1}$ are order-preserving.

This follows from the definition of $\phi$ and is left to the reader as an exercise.

\section{Weak subalgebra lattices}

In this section we generalize the result of K. Pióro 7 to the class of all finite groupoids and then we will show that the proof runs for any finite partial algebras, too.

Let $\mathcal{A}=\left(A ;\left(f_{t}^{(A)}\right)_{t \in T}\right)$ be a partial algebra of type $\tau$. We denote by $\emptyset(\mathcal{A})$ the ideal of the lattice $S_{w}(\mathcal{A})$ generated by the partial subalgebra

$$
\left(A ;\left(f_{t}^{(A)}\right)_{t \in T}\right) \quad \text { where } f_{t}^{(A)}=\emptyset \quad \text { for each } t \in T ;
$$

this partial subalgebra will be denoted simply as $(A ; \emptyset, \ldots, \emptyset)$. It follows that the ideal $\emptyset(\mathcal{A})$ is the Boolean lattice generated by the set of all atoms of the lattice $S_{w}(\mathcal{A})$.

Lemma 3.1. If a weak subalgebra $\mathcal{B}$ of $\mathcal{A}$ does not belong to the ideal $\emptyset(\mathcal{A})$ then there exists a join irreducible element $\mathcal{D} \in S_{w}(\mathcal{A})-\emptyset(\mathcal{A})$ of the lattice $S_{w}(\mathcal{A})$ such that $\mathcal{D} \leq \mathcal{B}$.

Pr o of. Since $\mathcal{B} \notin \emptyset(\mathcal{A})$, there is $t \in T$ with $f_{t}^{(B)} \neq \emptyset$. If $f_{t}^{(B)}\left(a_{1}, \ldots, a_{n\left(f_{t}\right)}\right)=a$, we can put $D=\left\{a_{1}, \ldots a_{n\left(f_{t}\right)}, a\right\}, f_{t}^{(D)}=\left\{\left[a_{1}, \ldots a_{n\left(f_{t}\right)}, a\right]\right\}$ and $f_{t^{\prime}}^{(D)}=\emptyset$ for all $t^{\prime} \in T \backslash\{t\}$.

COROLlary 3.2. Let $\mathcal{A}, \mathcal{A}^{\prime}$ be partial finite algebras of the same type. If there is an isomorphism $\phi$ of the lattice $S_{w}(\mathcal{A})$ onto $S_{w}\left(\mathcal{A}^{\prime}\right)$, then

$$
\phi(\emptyset(\mathcal{A}))=\emptyset\left(\mathcal{A}^{\prime}\right) \& \phi((A, \emptyset, \ldots, \emptyset))=\left(A^{\prime}, \emptyset, \ldots, \emptyset\right) .
$$

Pr o o f. The atoms of $S_{w}(\mathcal{A})$ are mapped in the isomorphism $\phi$ onto the atoms of $S_{w}\left(\mathcal{A}^{\prime}\right)$, hence also the Boolean lattice $\emptyset(\mathcal{A})$ generated by the set $A\left(S_{w}(\mathcal{A})\right)$ of all atoms of the lattice $S_{w}(\mathcal{A})$ is mapped onto the Boolean lattice $\emptyset\left(\mathcal{A}^{\prime}\right)$ generated by the set $A\left(S_{w}\left(\mathcal{A}^{\prime}\right)\right)$ of all atoms of the lattice $S_{w}\left(\mathcal{A}^{\prime}\right)$. 
Let $\mathcal{G}=(G, f)$ be a total groupoid. We denote by $S(\mathcal{G})$ the lattice of all its subgroupoids (therefore the subgroupoid $(\emptyset, \emptyset)$ is not included).

We shall show that for any finite groupoid $\mathcal{G}$, its weak subgroupoid lattice $S_{w}(\mathcal{G})$ uniquely determines its subgroupoid lattice $S(\mathcal{G})$.

Theorem 3.3. Let $\mathcal{G}=(G, f), \mathcal{G}^{*}=\left(G^{*}, f^{*}\right)$ be finite partial groupoids. If $\mathcal{G}$ is a total groupoid and there is an isomorphism $\phi$ of the lattice $S_{w}(\mathcal{G})$ onto the lattice $S_{w}\left(\mathcal{G}^{*}\right)$, then $\mathcal{G}^{*}$ is also a total groupoid and the restriction $\phi\lceil S(\mathcal{G})$ is an isomorphism of the lattice $S(\mathcal{G})$ to the lattice $S\left(\mathcal{G}^{*}\right)$, that is, the lattices of all total subgroupoids are isomorphic, too.

Pr o of. Evidently, $|G|=\left|A\left(S_{w}(\mathcal{G})\right)\right|=\left|A\left(S_{w}\left(\mathcal{G}^{*}\right)\right)\right|=\left|G^{*}\right|$ and so the isomorphism $\phi$ induces a bijection $\psi$ between the sets $G$ and $G^{*}$ given by

$$
\psi(a)=a^{\prime} \Longleftrightarrow \phi((\{a\}, \emptyset))=\left(\left\{a^{\prime}\right\}, \emptyset\right) .
$$

By Lemma 1.4, the groupoid $(G, \emptyset)$ can be in $S_{w}(\mathcal{G})$ covered only by elements $(G,\{[a, b, c]\})$ where $a, b, c \in G$ are not necessarily distinct, and the number of such triples $[a, b, c]$ equals the number of possible triples $f(a, b)=c$, and hence it is equal to $|G \times G|$. This yields that the groupoid $\mathcal{G}$ is total if and only if the element $(G, \emptyset)$ is covered in $S_{w}(\mathbf{G})$ by $|G \times G|$ elements; further, an element $\left(G^{\prime}, f^{\left(G^{\prime}\right)}\right)$ covers in $S_{w}(\mathcal{G})$ the element $(G, \emptyset)$ if and only if $G^{\prime}=G$ and $f^{\prime}=\{[x, y, z]\}$ where $f(x, y)=z$. As the lattice isomorphism preserves the covering relation, the element $\phi((G, \emptyset))=\left(G^{*}, \emptyset\right)$ is also covered in $S_{w}\left(\mathbf{G}^{*}\right)$ by $|G \times G|=\left|G^{*} \times G^{*}\right|$ elements of the above type. It follows that $\left(G^{*}, f^{*}\right)$ is a total groupoid, too.

Let $\mathcal{H}=(H, f)$ be a total subgroupoid of the groupoid $\mathcal{G}$ with $H \neq G$ and let $\phi((H, \emptyset))=\left(H^{\prime}, \emptyset\right)$. It is obvious that

$$
\phi((H, \emptyset))=\phi(\bigvee\{(\{d\}, \emptyset) \mid d \in H\})=\bigvee\left\{\left(\left\{d^{\prime}\right\}, \emptyset\right) \mid d^{\prime} \in H^{\prime}\right\}
$$

and so $|H|=\left|H^{\prime}\right|$. The subgroupoid $(H, \emptyset)$ is covered in the lattice $S_{w}(\mathcal{G})$ by $|H \times H|$ elements of the type $(H,\{[x, y, z]\})$ (where $f(x, y)=z$ and $x, y, z \in H)$. Then the element $\left(H^{\prime}, \emptyset\right)$ is covered in the lattice $S_{w}\left(\mathcal{G}^{*}\right)$ by $\left|H^{\prime} \times H^{\prime}\right|=|H \times H|$ elements. Therefore $\phi((H, f))=\left(H^{*}, f^{*}\right)$ is a total subgroupoid, too.

Theorem 3.4. Let $\mathcal{A}=\left(A, f_{1}^{(A)}, \ldots f_{n}^{(A)}\right), \mathcal{B}=\left(B, f_{1}^{(B)}, \ldots f_{n}^{(B)}\right)$ be finite partial algebras of the same finite type. If $\mathcal{A}$ is a total algebra and there is an isomorphism $\phi$ of the lattice $S_{w}(\mathcal{A})$ to the lattice $S_{w}(\mathcal{B})$, then $\mathcal{B}$ is also a total algebra and the restriction $\phi\lceil S(\mathcal{A})$ is an isomorphism of the lattice $S(\mathcal{A})$ to the lattice $S(\mathcal{B})$.

Pro of. Similarly as in the previous proof, $|A|=\left|A\left(S_{w}(\mathcal{A})\right)\right|=\left|A\left(S_{w}(\mathcal{B})\right)\right|$ $=|B|$ and $\phi((A, \emptyset, \ldots, \emptyset))=(B, \emptyset, \ldots, \emptyset)$. Let $\mathcal{A}$ be a total algebra. When 
$f_{1}^{(A)}=\{c\}$ is a nullary operation, the weak subalgebra $\mathcal{A}_{0}=(A, \emptyset, \ldots, \emptyset)$ is covered in the lattice $S_{w}(\mathcal{A})$ by an element $(A,\{c\}, \emptyset, \ldots, \emptyset)$. When $f_{1}^{(A)}$ is a unary operation, then $\mathcal{A}_{0}$ is covered by $|A|$ elements of the type $(A,\{[x, y]\}, \emptyset, \ldots, \emptyset)$. When $f_{1}^{(A)}$ is a binary operation then $\mathcal{A}_{0}$ is covered in the lattice $S_{w}(\mathcal{A})$ by $|A \times A|$ elements of the type $(A ;\{[x, y, z]\}, \emptyset, \ldots, \emptyset)$, etc. Thus, if $f_{i}$ is a $k_{i}$-ary operational symbol for each $i \in\{1, \ldots, n\}$, then the weak subalgebra $\mathcal{A}_{0}$ is covered in the lattice $S_{w}(\mathcal{A})$ by $|A|^{k_{1}}+|A|^{k_{2}}+\cdots+|A|^{k_{n}}$ elements which do not belong to the ideal $\emptyset(\mathcal{A})$. Therefore the element $\phi\left(A_{0}\right)$ is also covered by $|A|^{k_{1}}+|A|^{k_{2}}+\cdots+|A|^{k_{n}}$ elements which do not belong to the ideal $\emptyset(B)$. It yields that $\mathcal{B}$ is a total algebra, too.

Similarly we can carry on the rest of the previous proof. We are leaving it to the reader as an exercise.

\section{REFERENCES}

[1] BALCAR, B.- ŠTĚPÁNEK, P.: Set Theory, Academia, Praha, 1986 (Czech).

[2] BARTOL, W.: Weak subalgebra lattices, Comment. Math. Univ. Carolin. 31 (1990), 405-410.

[3] HALL, P.: On representatives of subsets, J. Lond. Math. Soc. (2) 10 (1935), 26-30.

[4] PIÓRO, K.: On a strong property of the weak subalgebra lattice, Algebra Universalis 40 (1998), 477-495.

[5] PIÓRO, K.: On connections between hypergraphs and algebras, Arch. Math. (Brno) 36 (2000), 45-60.

[6] PIÓRO, K.: The weak subalgebra lattice of a unary partial algebra of a given infinite unary type, Math. Slovaca 51 (2001), 295-320.

[7] PIÓRO, K.: On subgroupoid lattices of some finite groupoid, Acta Math. Univ. Comenian. (N.S.) LXXII (2003), 147-158.

Received 8. 7. 2013

Accepted 22. 11. 2013
Faculty of Natural Sciences

M. Bel University

Tajovského 40

SK-974 01 Banská Bystrica

SLOVAKIA

E-mail: alfonz@haviar.com miroslav.haviar@umb.sk 